\title{
Late Prosthetic Valve Endocarditis
}

National Cancer Institute

\section{Source}

National Cancer Institute. Late Prosthetic Valve Endocarditis. NCI Thesaurus. Code C128358.

Prosthetic valve endocarditis that occurs several months to years following valve replacement. 